\title{
Pregnancy Outcomes in COVID-19: A Prospective Cohort Study in Singapore
}

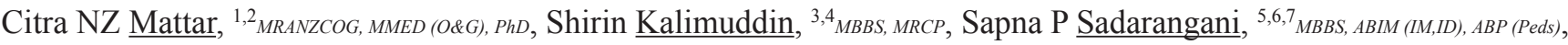
Shephali Tagore, ${ }^{8}$ MBBS, MD (O\&G), FRCOG, Serene Thain, ${ }^{8}$ MRCP, MRCOG, M Med (O\&G), Koh Cheng Thoon, ${ }^{9}$ MBBS, MRCPCH, MMed (Paeds), Eliane Y $\underline{\text { Hong, }}{ }^{2} M B C h B, M R C O G$, Abhiram Kanneganti, ${ }^{2} M B B S$, MRCS, Chee Wai Ku,,${ }^{10}{ }_{M D, M R C O G, M M E D}(O \& G)$, Grace MF Chan, ${ }^{2} M B B S$, Kelvin ZX Lee, ${ }^{2} M D$, Jeannie JY Yap, ${ }^{2}$ MBChB, Shaun S Tan, ${ }^{11}{ }_{M B C h B, ~ M R C P S G,} M S$, Benedict Yan, ${ }^{11}{ }_{M B B S,}$ FRCPath, Barnaby E Young, ${ }^{5,6,7}$ MB BChir, MRCP, DLSHTM, David C Lye, ${ }^{5,6,7,12}$ MBBS, FRACP, FRCP, Danielle E Anderson, ${ }^{4}{ }_{P h D}$,

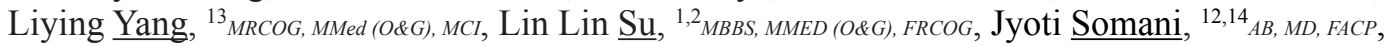

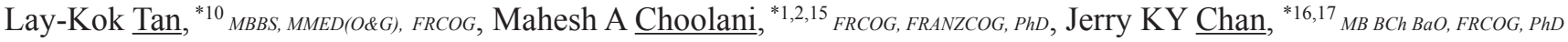

\begin{abstract}
Introduction: Pregnant women are reported to be at increased risk of severe coronavirus disease 2019 (COVID-19) due to underlying immunosuppression during pregnancy. However, the clinical course of COVID-19 in pregnancy and risk of vertical and horizontal transmission remain relatively unknown. We aim to describe and evaluate outcomes in pregnant women with COVID-19 in Singapore.

Methods: Prospective observational study of 16 pregnant patients admitted for COVID-19 to 4 tertiary hospitals in Singapore. Outcomes included severe disease, pregnancy loss, and vertical and horizontal transmission.

Results: Of the 16 patients, $37.5 \%, 43.8 \%$ and $18.7 \%$ were infected in the first, second and third trimesters, respectively. Two gravidas aged $\geq 35$ years (12.5\%) developed severe pneumonia; one patient (body mass index $32.9 \mathrm{~kg} / \mathrm{m}^{2}$ ) required transfer to intensive care. The median duration of acute infection was 19 days; one patient remained reverse transcription polymerase chain reaction (RT-PCR) positive $>11$ weeks from diagnosis. There were no maternal mortalities. Five pregnancies produced term live-births while 2 spontaneous miscarriages occurred at 11 and 23 weeks. RT-PCR of breast milk and maternal and neonatal samples taken at birth were negative; placenta and cord histology showed non-specific inflammation; and severe acute respiratory syndrome coronavirus 2 (SARS-CoV-2)-specific immunoglobulins were elevated in paired maternal and umbilical cord blood $(n=5)$.

Conclusion: The majority of COVID-19 infected pregnant women had mild disease and only 2 women with risk factors (obesity, older age) had severe infection; this represents a slightly higher incidence than observed in age-matched non-pregnant women. Among the women who delivered, there was no definitive evidence of mother-to-child transmission via breast milk or placenta.
\end{abstract}

Ann Acad Med Singap 2020;49:857-69

Keywords: Pregnancy outcomes, maternal morbidity, mother-child transmission, SARS-CoV-2, transferred immunity

\footnotetext{
${ }^{1}$ Department of Obstetrics and Gynaecology, Yong Loo Lin School of Medicine, National University of Singapore, Singapore

${ }^{2}$ Department of Obstetrics and Gynaecology, National University Hospital, Singapore

${ }^{3}$ Department of Infectious Diseases, Singapore General Hospital, Singapore

${ }^{4}$ Programme in Emerging Infectious Diseases, Duke-NUS Medical School, Singapore

${ }^{5}$ National Centre for Infectious Diseases, Singapore

${ }^{6}$ Department of Infectious Diseases, Tan Tock Seng Hospital, Singapore

${ }^{7}$ Lee Kong Chian School of Medicine, Nanyang Technological University, Singapore

${ }^{8}$ Department of Maternal Fetal Medicine, KK Women's and Children's Hospital, Singapore

${ }^{9}$ Infectious Disease Service, Paediatrics, KK Women's and Children's Hospital, Singapore

${ }^{10}$ Division of Obstetrics and Gynaecology, KK Women's and Children's Hospital, Singapore

${ }^{11}$ Laboratory Medicine, National University Hospital, Singapore

${ }^{12}$ Yong Loo Lin School of Medicine, National University of Singapore, Singapore

${ }^{13}$ Department of Obstetrics and Gynaecology, Singapore General Hospital, Singapore

${ }^{14}$ Division of Infectious Diseases, Department of Medicine, National University Hospital, Singapore

${ }^{15}$ Department of Obstetrics and Gynaecology, National University Health System, Singapore

${ }^{16}$ Department of Reproductive Medicine, KK Women's and Children's Hospital, Singapore

${ }^{17}$ Programme in Cancer and Stem Cells Biology / Obstetrics \& Gynaecology Academic Clinical Program, Duke-NUS Medical School, Singapore Addresses for Correspondence:

Prof Jerry KY Chan, KK Women's and Children's Hospital, 100 Bukit Timah Road, Singapore 229899. Email: jerrychan@duke-nus.edu.sg

A/Prof Citra NZ Mattar, National University of Singapore, NUHS Tower Block, Level 12, 1E Kent Ridge Road, Singapore 119228. Email: citramattar@nus.edu.sg

*Joint senior authors
} 


\section{Introduction}

Since the first cases of coronavirus disease 2019 (COVID-19) in pregnancy were described, ${ }^{1}$ significant concerns have been raised about the potentially increased susceptibility of pregnant women to severe disease, ${ }^{2}$ and the unquantified risk of mother-child transmission of severe acute respiratory syndrome coronavirus 2 (SARS-CoV-2) to the fetus and neonate. ${ }^{3}$ Despite our experience with other $\beta$ coronavirus infections in pregnancy, including severe acute respiratory syndrome (SARS) and Middle East respiratory syndrome (MERS), ${ }^{4}$ many questions remain regarding the clinical course of COVID-19 in pregnancy. A case series of 116 cases from Wuhan, China, reported a $6.9 \%$ incidence of severe pneumonia but no mortality among infected pregnant women, in contrast to the case fatality rate of $1-3 \%$ in the general population. ${ }^{5}$ Earlier case series from Italy, the US and Sweden also reported no maternal mortalities and critical care admission in $<10 \%$ of infected gravidas, ${ }^{6}$ findings similar to systematic reviews involving $>160$ pregnancies that described favourable maternal and fetal outcomes, possibly related to pregnancy-specific physiological changes that mitigate COVID-19 severity. ${ }^{2}$ More recently, however, there has been growing recognition of unexpected maternal mortality and severe morbidity associated with respiratory and thromboembolic complications affecting both low- and high-resource countries, including 15 reported fatalities in Iran, Brazil and Mexico, and at least 3 in the UK and US. ${ }^{7,8}$ Additionally, the unquantified risks of fetal SARS-CoV-2 infection increase the complexities of prenatal and perinatal management. The evidence for vertical transmission is still inconclusive. Published case series have reported mixed evidence of viral transmission via transplacental, vaginal or breastmilk routes, although the majority of these infections occurred in the late third trimester when the duration of viral exposure was limited. ${ }^{9}$ There is currently insufficient evidence to quantify the risks of vertical transmission when infection occurs at earlier gestations. ${ }^{10}$ Here, we present our experience managing 16 pregnant women diagnosed with COVID-19 in all trimesters in Singapore. We discuss the range of clinical manifestations, including trends towards a higher incidence of severe disease compared to age-matched non-pregnant women, and comprehensive analyses of perinatal samples in a subset of postpartum patients that have excluded vertical and horizontal transmission in our cohort so far.

\section{Methods}

\section{Study participants}

In this nationwide, prospective, multicentre study, we included all pregnant women with COVID-19 diagnosed by reverse transcription polymerase chain reaction (RT-PCR), who were admitted between 15 March 2020 and 22 August 2020 to the National University Hospital, KK Women's and Children's Hospital (KKH), Singapore General Hospital, and the National Centre for Infectious Diseases, all of which were the national receiving centres for COVID-19 maternal infections in Singapore.

\section{Ethics approval}

Ethics approval was obtained from National Healthcare Group Domain Specific Review Board. Verbal and written consent was obtained for collection of biological samples for clinical investigations. Informed consent was waived for collection of clinical data under the Infectious Diseases Act (Ministry of Health, Singapore) as part of COVID-19 pandemic investigations. Procedures were followed in accordance with the Declaration of Helsinki (1964, amended 2008) of the World Medical Association.

\section{Screening and diagnosis}

Pregnant women with acute respiratory symptoms, who had contact with known COVID-19 cases, had significant travel history, or were exposed to known community clusters, were screened with nasopharyngeal swabs. Only medically indicated, and not universal, screening was performed in Singapore during that time. Diagnosis of SARS-CoV-2 infection was made by RT-PCR of viral nucleic acids utilising the fully automated cobas ${ }^{\circledR}$ SARS-CoV-2 test on the cobas ${ }^{\circledR} 6800$ Systems (Roche Molecular Systems, Branchburg, US), with selective amplification of ORF1, a non-structural region of the coronavirus genome unique to SARS-CoV-2, with target-specific forward and reverse primers, in line with World Health Organization recommendations. ${ }^{11}$ Patients were isolated in negative pressure rooms until the diagnosis was confirmed and thereafter transferred to hospitals with maternity services where they were nursed in dedicated isolation wards. Patients were discharged after complete symptom resolution and 2 consecutive negative RT-PCR respiratory samples obtained 24 hours apart, or after at least 21 days had passed from symptom onset, according to updated Ministry of Health advisories (Fig. 1). 


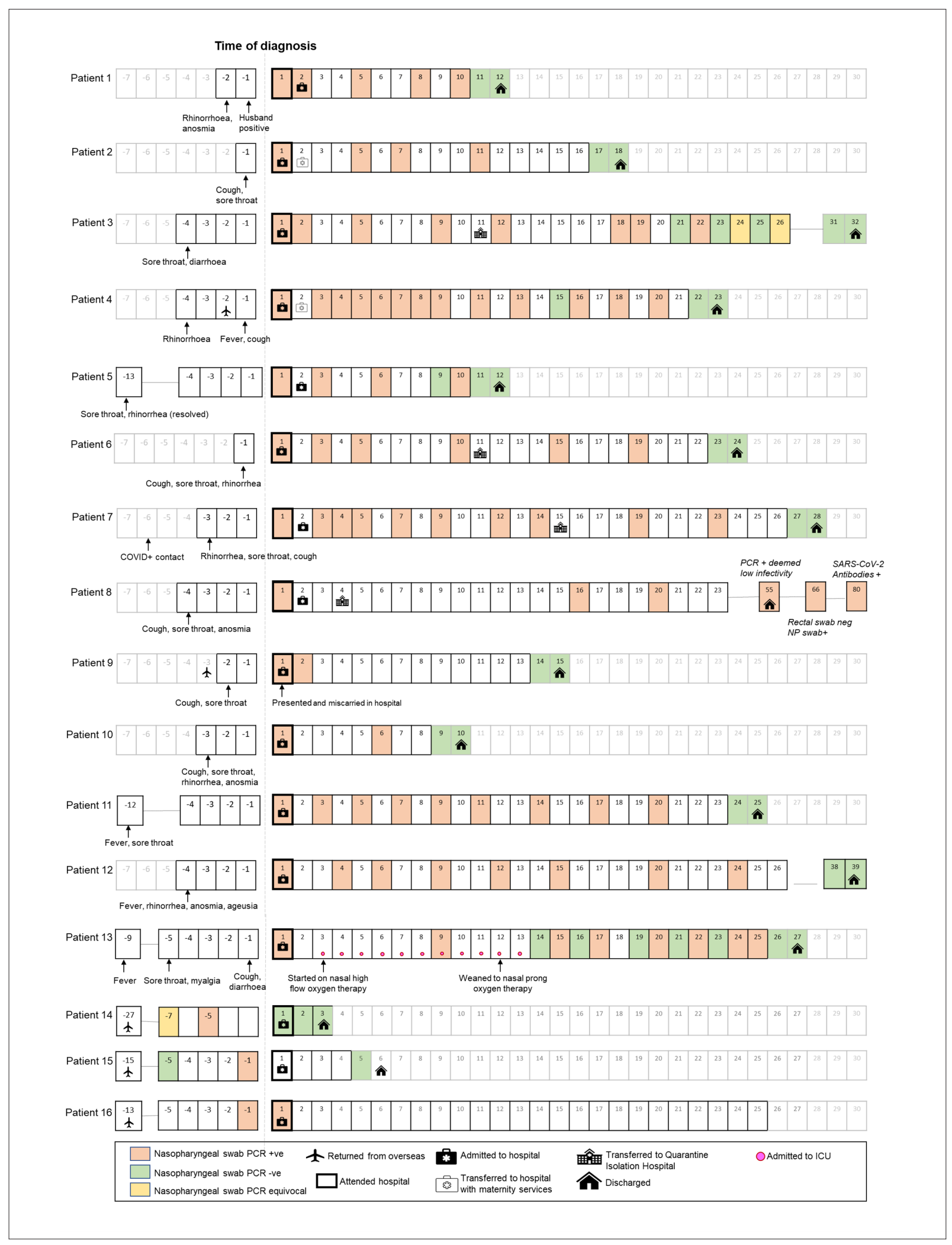

Fig. 1. Timeline for each patient from symptom onset to convalescence. Boxes represent days numbered in reference to the day of admission and are coloured according to nasopharyngeal swab reverse transcription polymerase chain reaction (RT-PCR) results for severe acute respiratory syndrome coronavirus 2 (SARS-CoV-2). Symbols are used to indicate significant events: hospital admission, intensive care unit transfer, transfer to the quarantine isolation hospital and discharge following 2 consecutive negative nasopharyngeal swabs at least 24 hours apart. Patients 8 and 16 remain positive at the time of writing. 


\section{Data and sample collection}

We obtained demographic, clinical, laboratory and epidemiological data from hospital electronic medical records. Longitudinal data collected included range and duration of presenting complaints, contact history, comorbidities, antenatal history, and pregnancy outcomes including fetal loss, fetal growth and perinatal outcomes. Routine baseline screening included complete blood counts, C-reactive protein (CRP) levels, and liver and renal biochemistry; these were repeated when clinically indicated. Fetal and maternal surveillance during acute infection and convalescence were enhanced to detect adverse effects of COVID-19, if any, and in selected cases included maternal rectal swabs for RT-PCR, and prenatal and postnatal administration of the Edinburgh Postnatal Depression Scale to screen for depression and anxiety. Fetal structural and growth scans were performed at 18, 20-22, 28-32 and 34-36 weeks' gestation. At delivery, perinatal samples were collected to assess for vertical transmission, including maternal blood and vaginal swabs, amniotic fluid and umbilical cord blood (UCB), and swabs of the placental and umbilical cord surfaces, all for SARS-CoV-2 RTPCR. Placenta and umbilical cord were histologically examined. Maternal and UCB sera were tested for SARS-CoV-2-specific immunoglobulins using the Elecsys Anti-SARS-CoV-2 assay (Roche Diagnostics, Basel, Switzerland), a sandwich immunoassay utilising a recombinant protein representing the nucleocapsid (N) antigen, performed after successful calibration and quality control. A signal cut-off index $(\mathrm{COI}) \geq 1.0$ indicated seroreactivity, while $\mathrm{COI}<1.0$ was seronegative.

\section{Statistical analyses}

Statistical analysis was conducted using GraphPad Prism version 8.4.2 for Windows (GraphPad Software, San Diego, US) with categorical variables expressed as percentage, and continuous variables expressed as a median (range). Comparison of outcomes was performed with Fisher's Exact test $(\mathrm{p}<0.05)$.

\section{Results}

Sixteen pregnant women with COVID-19 were included in this study (Table 1). Maternal age ranged from 23-36 years. Diverse ethnicities were represented (Malay, $n=5$; Chinese, $n=3$; Indian, $n=4$; Eurasian, $n=1$; Caucasian, $\mathrm{n}=3$ ). Gestations at diagnosis ranged from 4 weeks (based on the last menstrual period) to 36 weeks, on ultrasound survey at admission (first trimester, $n=6$; second trimester, $n=7$; third trimester, $n=3$ ). Three patients had significant comorbidities: patient 6 had gallstone disease and was a hepatitis $\mathrm{C}$ carrier with normal levels of transaminases; patients 8 and 14 had well-controlled asthma; and the other patients had no significant comorbidities. Three patients (18.8\%) were asymptomatic, while $13(81.2 \%)$ had mild respiratory symptoms on initial assessment at admission, including cough, sore throat, rhinorrhoea, anosmia and ageusia. No patients diagnosed with COVID-19 in the second or third trimesters developed obstetric complications; $87.5 \%(n=14)$ had mild disease, remained afebrile throughout admission, and did not require supplemental oxygen. The exceptions were 2 women (Patients 9 and 13) who had complicated clinical courses, described separately below.

Two women diagnosed in their first trimester of pregnancy were admitted with their infant children who were breastfeeding and also COVID-19 positive, and 2 women in their third trimester were transferred to the same hospital units as their COVID-19 positive partners, following a national policy of keeping families together. Daily physical examination of the cardiorespiratory system and biweekly fetal heart rate examination (in viable pregnancies $>24$ weeks of gestation) were performed. Women in the first trimester did not routinely undergo viability assessment unless they reported bleeding with or without pelvic pain; ultrasound assessments were performed once the woman was RT-PCR negative for SARS-CoV-2, a policy effected to mitigate risk to medical staff. Symptomatic treatment was prescribed for upper respiratory tract symptoms and fever. Multidisciplinary management was provided by infectious disease and maternal-fetal specialists, with additional input from respiratory physicians and psychological support teams (psychiatrists and counsellors) where indicated. Chest radiographs (CXR) were clinically indicated in patients 9, 11, 12 and 13 for persistent fever, despite the initial lung examination being clear in the first 3 patients. The CXR were performed on patients 11 and 12 before their early pregnancies were diagnosed, and these were normal. Some women who were clinically stable with mild or no symptoms, but were still RT-PCR positive, were transferred to a community isolation facility and remained there until they were negative on RT-PCR following national policy. The clinical course from admission to discharge is summarised in Fig. 1.

Nine patients $(56.2 \%)$ had raised absolute neutrophil count or CRP levels, or both, while only patient 8 had lymphopaenia $\left(0.8 \times 10^{9}\right.$ cells $\left./ \mathrm{L}\right)$ and monocytopenia $\left(0.2 \times 10^{9}\right.$ cells/L) (Fig. 2A). Anaemia was observed in 4 $(23 \%)$ and ferritin levels were low in $2(12.5 \%)$; renal 


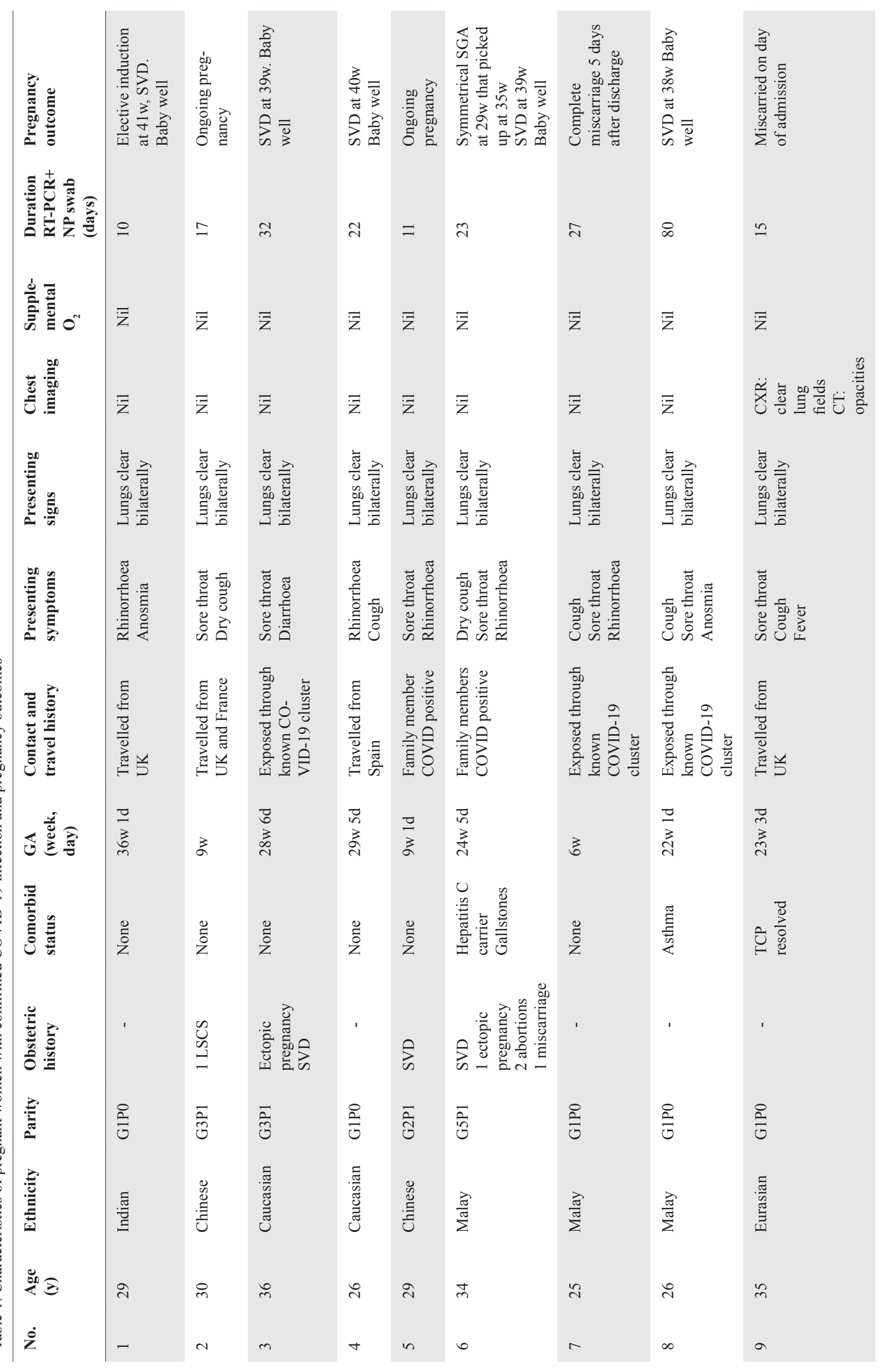




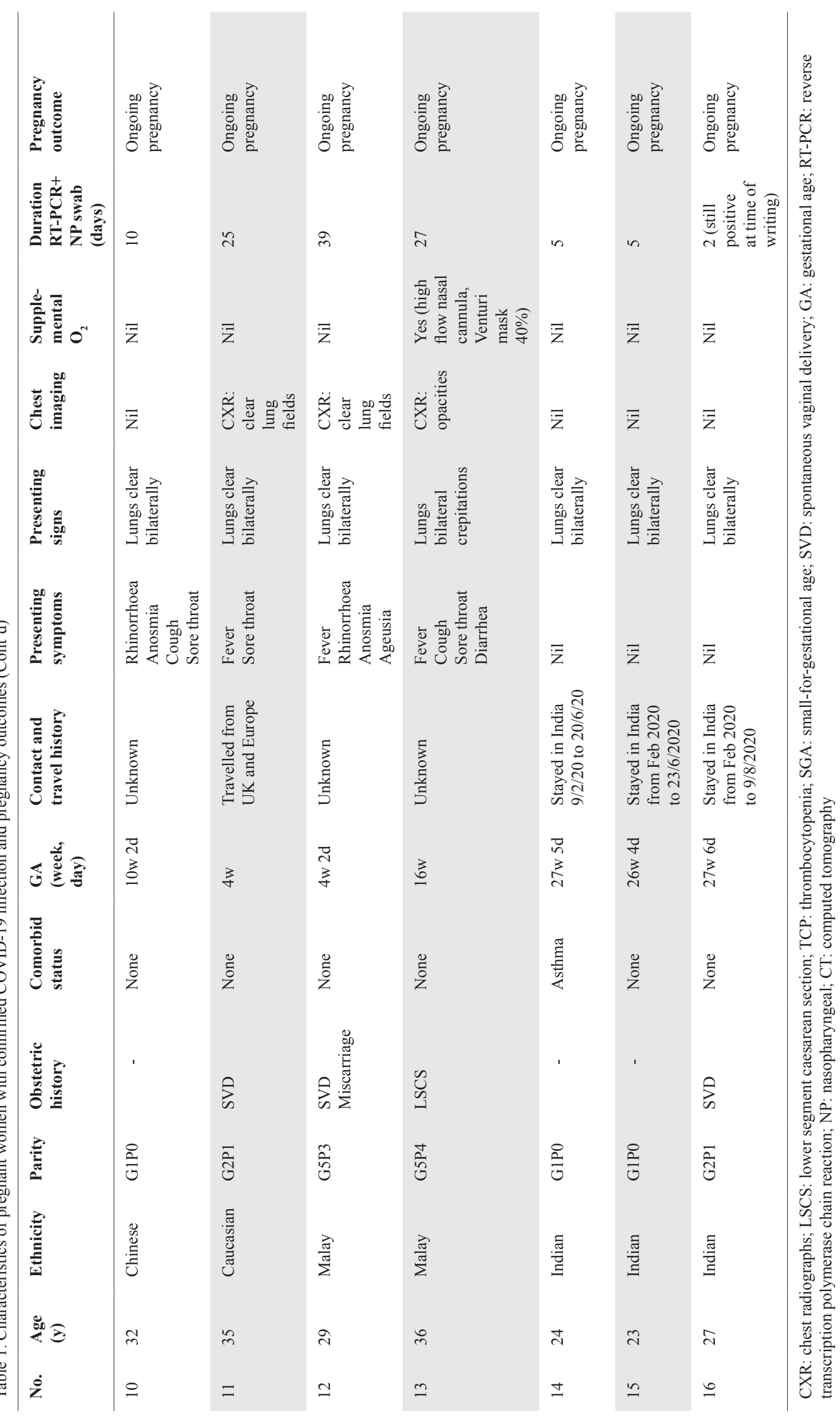




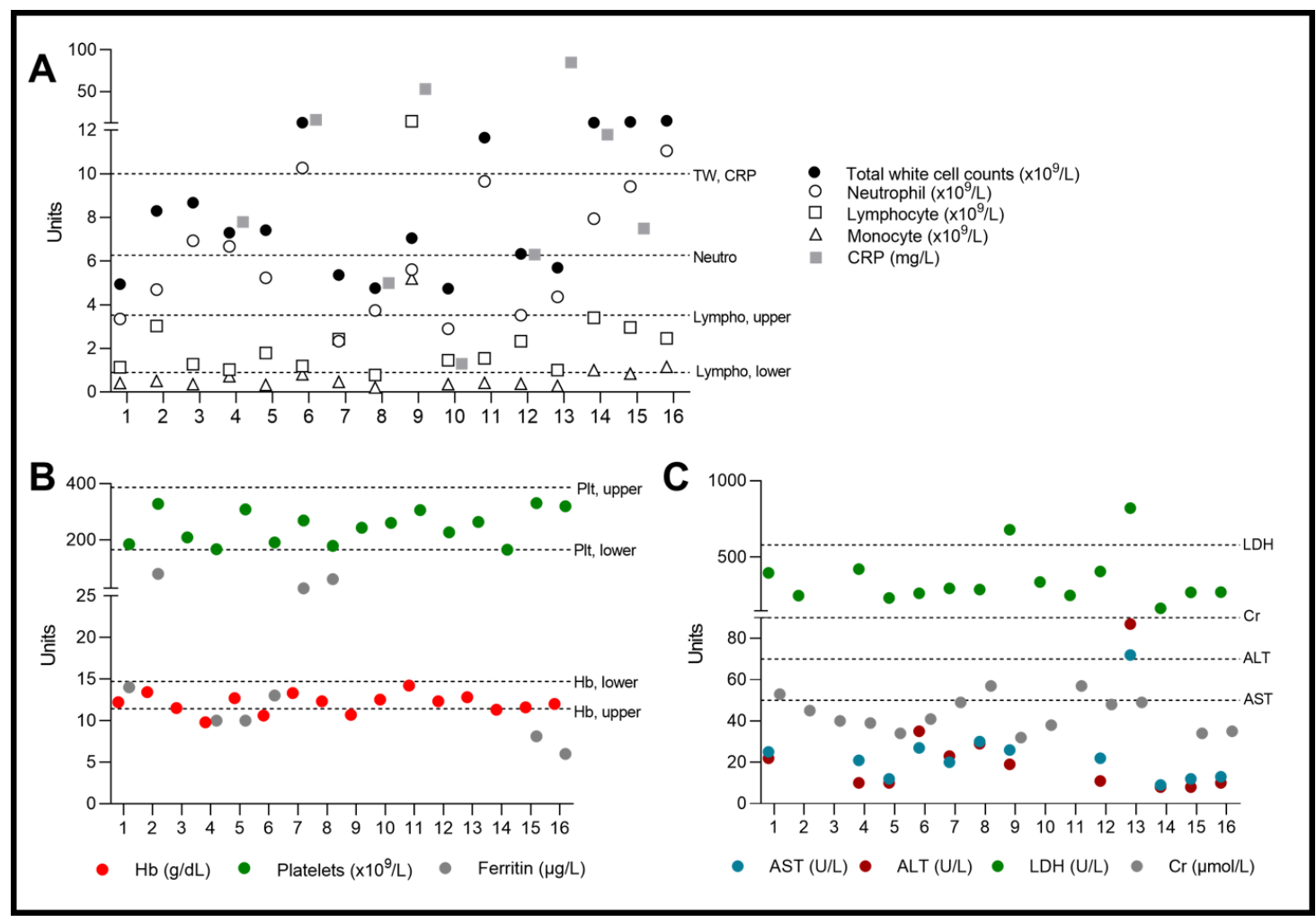

Fig. 2. Biochemistry results for patients 1 to 16 (x-axis) on admission. (A) Patients 9 and 13 who developed severe COVID-19 infection had raised C-reactive protein (CRP) and lactate dehydrogenase (LDH) levels. (B) Haematological indices for all patients were largely normal. (C) Patient 13 had raised aspartate aminotransferase (AST) and alanine aminotransferase (ALT) levels which normalised before discharge.

and liver functions were normal in all patients at presentation (Fig. 2B, 2C). The median duration of viral shedding, from the first RT-PCR positive nasopharyngeal swab to the second RT-PCR negative result (or the last documented swab test), was 19 days (range, 2-80 days). Ten patients $(62.5 \%)$ received stress management education from peer counsellors during hospitalisation and $2(12.5 \%)$ were prescribed anti-anxiety medications by a psychiatrist. Five patients consented to rectal swabs for RT-PCR during convalescence to assess continued SARS-CoV-2 shedding, all of which were negative. Fourteen patients $(87.5 \%)$ have been discharged at the time of writing. One patient remained admitted while awaiting criteria for discharge. The other patient remained RT-PCR positive 80 days after development of initial symptoms but was deemed no longer at risk of secondary transmission and discharged following a national policy shift from test-based to time-based de-isolation; ${ }^{12}$ she also displayed seropositivity (COI, 13.5) despite the positive RT-PCR (Fig. 1). There were no maternal mortalities.

Patient 9 (35 years old) received her initial antenatal care in the UK and reported a low-risk first-trimester fetal aneuploidy screen and normal fetal anatomy survey at 21 weeks' gestation. She had multiple uterine fibroids (including a $9.5 \mathrm{~cm}$ cervical fibroid). She presented to KKH 3 days after arriving from the UK with a recent onset of cough, chest discomfort and lower abdominal pain at 23 weeks 3 days of gestation. Her partner also reported a productive cough. They had no contact with suspected or confirmed COVID-19 cases. On presentation, the patient was febrile $\left(38.9^{\circ} \mathrm{C}\right)$, tachypnoeic (20 breaths/ min), normotensive and had clear lung fields with no crepitations or rhonchi. There was no uterine tenderness indicative of red degeneration of the fibroid. Her oxygen saturations remained at $>94 \%$ and she did not require supplemental oxygen. Two consecutive nasopharyngeal swabs were positive for SARS-CoV-2 on RT-PCR. The patient developed painful contractions soon after admission and miscarried approximately 12 hours later. Although the initial CXR was normal, computed tomography of the thorax was performed the following day for persistent postnatal fever, and showed multiple bilateral ground-glass opacities, predominantly subpleural and in the lower pulmonary lobes, some of which demonstrated increased peripheral density (reversed 
halo sign; Fig. 3A-3D). Maternal serum and urine, swabs of the fetal ear, nasopharynx and oropharynx, fetal cord blood, and placenta surfaces (fetal and maternal) were negative on RT-PCR for SARS-CoV-2. She was transferred to Singapore General Hospital for further management and received intravenous antibiotics until blood cultures returned negative. She maintained good oxygen saturation levels without the need for supplemental oxygen, remained haemodynamically stable throughout her admission, and was discharged clinically well after 15 days.

Patient 13 (36 years old; body mass index $32.9 \mathrm{~kg} /$ $\mathrm{m}^{2}$ ) presented with generalised myalgia and lethargy ( $>7$ days), and fever, dry cough and sore throat (1 day) at 18 weeks 4 days of gestation. She was febrile $\left(38.3^{\circ} \mathrm{C}\right)$, tachycardic $(120$ beats $/ \mathrm{min})$ and had bilateral pulmonary crepitations on chest auscultation with an initial oxygen saturation of $95 \%$ on room air, as measured by pulse oximetry. The CXR demonstrated bilateral airspace opacities (Fig. 3E, 3F). The patient's clinical condition worsened over the next 48 hours and she was subsequently transferred to the intensive care unit (ICU) because of an increasing need for support with high flow oxygen. She did not require intubation but remained in ICU for 10 days until being weaned off supplemental oxygen. Transient transaminitis was observed on day 3 of admission (aspartate aminotransferase, 72U/L; alanine aminotransferase, 87U/L; lactate dehydrogenase, $822 \mathrm{U} / \mathrm{L}$ ) and normalised after 19 days (Fig. 2C). Following discharge on day 25 (22 weeks 2 days of gestation) when she was clinically well, the fetal structural ultrasound survey showed normal anatomy and growth.

Of the 9 patients with previable pregnancies $(<24$ weeks' gestation), 2 (22.2\%) had spontaneous miscarriages. Patient 7 presented with painless vaginal bleeding at 11 weeks amenorrhoea, 5 days after discharge. Serial transvaginal ultrasonography and serum beta human chorionic gonadotropin assays confirmed an early miscarriage at home which was managed expectantly. Clinical surveillance retrospectively confirmed spontaneous complete pregnancy resolution and no products of conception were collected for RT-PCR. Patient 9 had a mid-trimester miscarriage as described above. Patient 6 who was diagnosed with COVID-19 at about 25 weeks' gestation was diagnosed with a symmetrically small-for-gestational-age fetus at 29 weeks (estimated fetal weight at the sixthcentile; normal amniotic fluid index and umbilical artery Doppler studies). Fetal growth had normalised by 35 weeks and she subsequently had an uncomplicated term vaginal delivery of a normal-birth-weight infant at 39 weeks. There were 4 other live births in this cohort. Patients 1, 3, 4 and 8 had otherwise uncomplicated pregnancies with reassuring fetal growth on surveillance, delivering vaginally at 39-41 weeks' gestation, 3 to 11 weeks after the last negative RT-PCR for SARS-CoV-2. In the 5 women who delivered, swabs of the vagina, umbilical cord, maternal and fetal placental surfaces, amniotic fluid, and maternal blood and UCB were negative for SARS-CoV-2 on RT-PCR, and histological examination of the placenta and umbilical cord did not reveal ischaemia, necrosis or funisitis (Table 2). While patient 1 had insufficient colostrum to screen for SARS-CoV-2 prior to discharge, colostrum from patients 4 and 6 was negative on RT-PCR. All mother-baby pairs had elevated SARS-CoV-2 total immunoglobulins. In patient 1 , perinatal maternal blood (COI 27.9) and UCB (COI 11.2) were strongly positive. Maternal blood and UCB immunoglobulins in patient 4 were mildly elevated in comparison (COI 1.5 and 2.8, respectively; Table 2). All mothers practised immediate skin-to-skin contact and direct breastfeeding, and mother-baby pairs were discharged well on postnatal day 2 .

\section{Discussion}

Most patients in our study had a mild clinical course, but 2 women developed severe pneumonia. Recent reports have highlighted the unpredictable clinical course of COVID-19 infection in pregnancy. ${ }^{13,14}$ Severe maternal disease can manifest prenatally or postnatally and trigger abrupt postnatal decompensation, and its presentation may be delayed up to 14 days from symptom onset. Patient 9 had no underlying comorbidities predictive of clinical deterioration. ${ }^{15}$ Her clinical status worsened precipitously following delivery, reflecting aggressive disease progression. Patient 13 was obese and both patients 9 and 13 were $\geq 35$ years old; obesity and older age have both been identified as risk factors in maternal deaths, along with diabetes mellitus, cardiorespiratory disease or thromboembolic complications. ${ }^{14}$ The median duration of viral shedding in our study was 19 days, although one patient remained positive up to 80 days after initial symptoms. Cohort studies have reported prolonged shedding up to 60 days; putative risk factors for this phenomenon were not present in this patient. ${ }^{16}$ Faecal excretion of SARS-CoV-2 for several weeks is well documented, raising concerns of neonatal transmission during vaginal delivery. ${ }^{17}$ Rectal swabs were obtained from some of our patients to inform labourmanagement, and all were negative. The mid-trimester pregnancy loss (patient 9) may be partly related to the severe systemic inflammatory response of the acute infection, although there is a paucity of immunological data to prove this. ${ }^{18}$ Similarly, with a population incidence of small-for- 


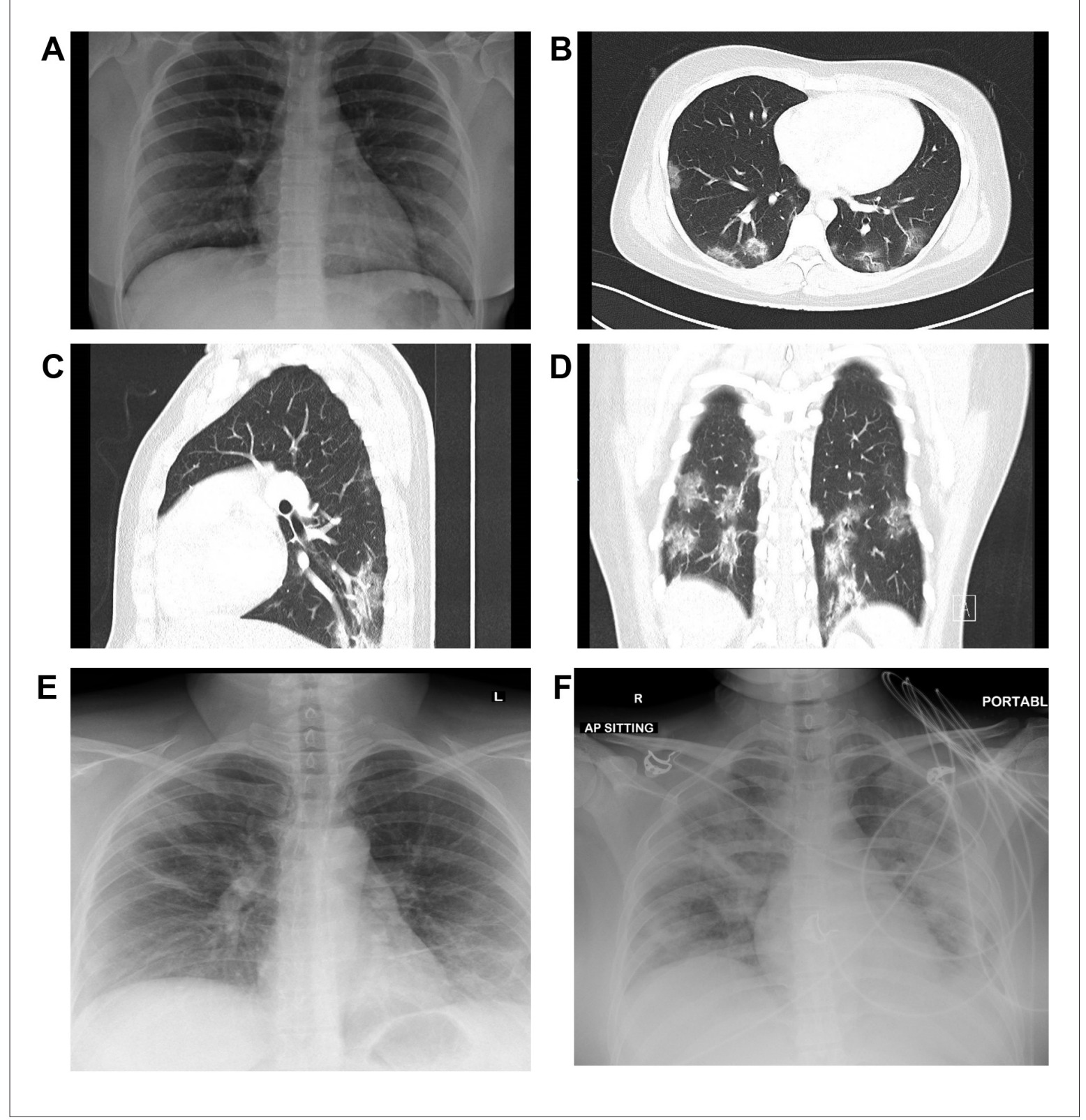

Fig. 3. (A) Chest X-ray for patient 9 performed on the day of admission did not show confluent consolidation or pleural effusion. (B, C) Computed tomography (CT) of the chest performed on the second day showed multiple ground-glass opacities in the peripheries of both lungs, predominantly subpleural and in the lower lobes, with someopacities demonstrating a reversed halosign.(D) Somedenseropacities areperibronchial inlocation, seen as an opacity in the posterior basal segment of the left lower lobe. (E)A chest X-ray for patient 13 performed on the day of admission showed streaky airspace opacities in the right upper to middle zone and left middle to lower zones. $(\mathrm{F})$ Asecond chest X-ray repeated on day 8 of admission showed interval worsening in bilateral lung consolidation with patchy involvement of both lungs.

gestational-age of $3-10 \%,{ }^{19}$ the growth restriction in patient 6 cannot be directly attributable to COVID-19. From the systematic screening of perinatal samples, we conclude that there is no evidence thus far of maternal-child transmission in our cohort following second and third trimester infections. Outcomes of first trimester infections are pending at the time of writing.

Peak community transmission of SARS-CoV-2 occurred in Singapore in March 2020 and drastically fell following strict government measures in April 2020. ${ }^{12}$ Our case series and others demonstrate that the incidence and severity of COVID-19 among pregnant women parallel the general population trend..$^{13,20}$ During this period, 256 patients aged between 21 and 35 years were admitted with virologically confirmed COVID-19 (up to 31 March 2020) in Singapore. Non-pregnant females comprised 41.4\% (106 of 256) of admissions, 14 of whom developed pneumonia with 1 patient requiring 


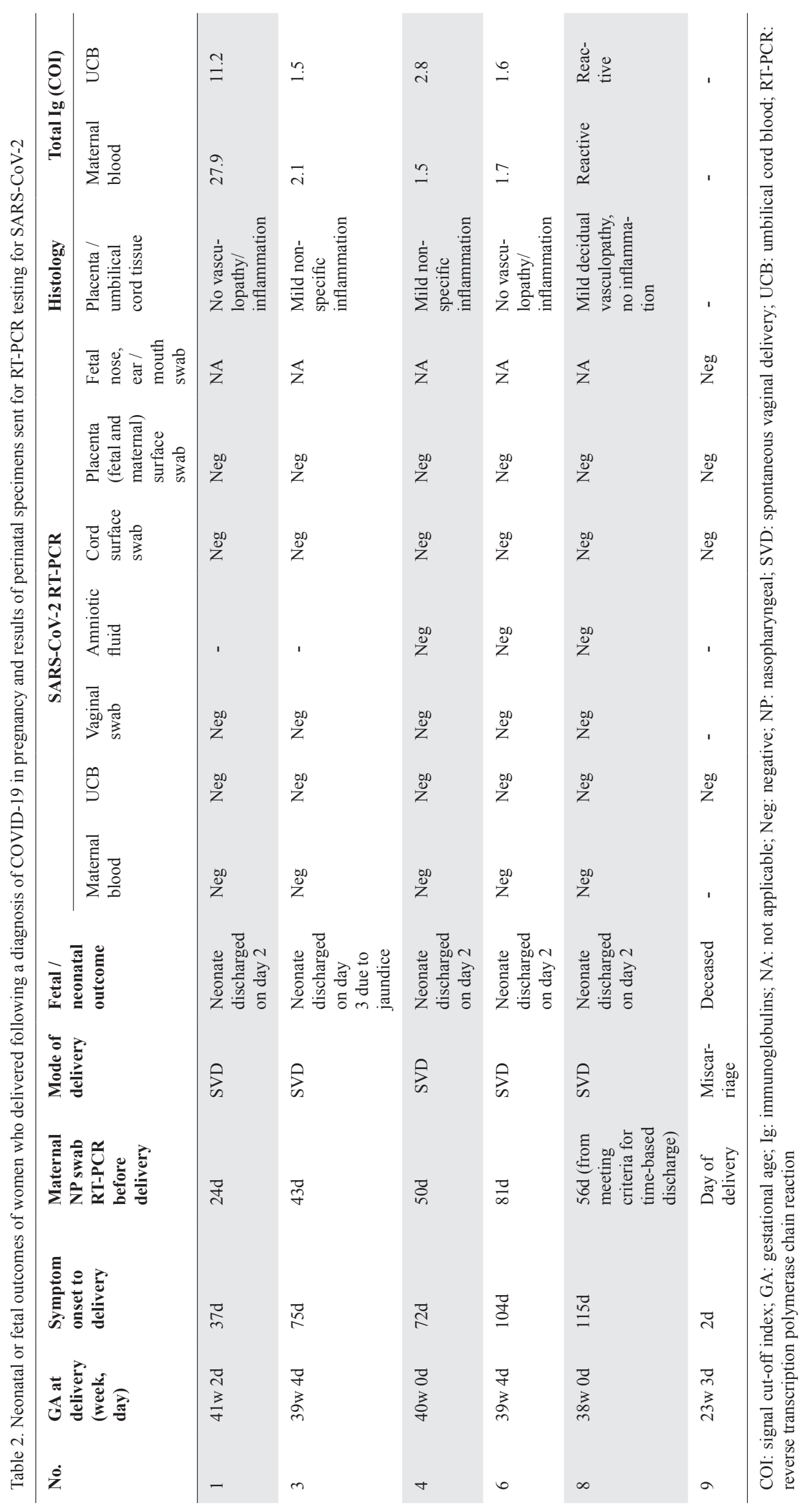


supplemental oxygen. While there was no difference between pregnant and non-pregnant females developing severe pneumonia $(15.4 \%$ vs. $13.2 \%$; $\mathrm{p}=0.69)$, a trend towards a higher incidence of supplemental oxygen use was observed among pregnant women $(7.7 \%$ vs. $0.9 \% ; P=0.21$ ).

The possibility of placental transmission of SARS$\mathrm{CoV}-2$ remains controversial. In our study, all perinatal tissue specimens were RT-PCR negative. In other studies, SARS-CoV-2 has been identified within the placenta using RT-PCR, viral genome sequencing, immunohistochemistry and electron microscopy, despite RT-PCR being negative for vaginal secretions, amniotic fluid, UCB and fetal tissues. ${ }^{21}$ Caution is advised with these methods as artefacts and other inclusions mimicking viral particles and non-specific staining decrease specificity. ${ }^{22}$ In addition, studies of placental histology have concluded that placental changes in women with COVID-19 were more likely related to maternal infection and inflammation, rather than fetal infection. ${ }^{18}$ Neonatal SARS-CoV-2-specific immunoglobulin $\mathrm{M}$ (IgM) may not be conclusive evidence of fetal seroconversion owing to technical limitations and possible transplacental trafficking of maternal IgM when the maternal-fetal interface is breeched by inflammation and hypoxia. ${ }^{23}$ Evidence in favour of mother-child transmission includes reports of 2 mother-infant pairs who demonstrated positive RT-PCR for SARS-CoV-2 on swabs of maternal and neonatal nasopharynx and from the fetal surface of the placenta, ${ }^{24}$ and a neonate delivered by caesarean section following maternal COVID-19 infection who then developed neurological manifestations at postnatal day 3. The latter describes multiple positive RT-PCR from placenta, nasopharynx, sera, vagina and pre-membrane rupture amniotic fluid, with a viral load much higher in placental tissue than in maternal blood or amniotic fluid, suggesting congenital COVID-19 infection. ${ }^{25}$ The main challenge in confirming vertical transmission is related to establishing mechanism, particularly as SARS-CoV-2 cell entry requires colocalisation of angiotensin-converting enzyme 2 and transmembrane protease serine 2 receptors, which are present in negligible levels in placentas. ${ }^{\text {? }}$

Our perinatal outcomes reflect the results of a systematic review reporting that the majority of neonates born to mothers with COVID-19 were healthy at birth. Neonates who were symptomatic developed pneumonia or non-specific features, including fever, tachycardia, thrombocytopenia and deranged liver function. The one mortality involved a preterm neonate who developed refractory shock, disseminated intravascular coagulation and multi-organ failure. Neonatal COVID-19 infection could not be definitively ruled out. At present, we and other researchers have no evidence of COVID-19 teratogenicity following infection in early gestation. While the breast milk from 2 postnatal patients in our cohort was RT-PCR-negative, serial screening may be required to establish this route of transmission following a minimal period of viraemia. ${ }^{26}$ All 4 delivered patients demonstrated a range of seropositivity in maternal blood and UCB atdelivery. Given the absence of viral RNA in UCB and extra-fetal tissues, this finding likely reflects transplacentally trafficked maternal immunoglobulin G. Patient 1 with higher immunoglobulin levels had a shorter infection-todelivery interval ( 37 vs. 72 days in patient 4); the lower $\mathrm{COI}$ in the other 3 patients may reflect natural maternal antibody decomposition during the longer convalescence. ${ }^{27}$ Patient 8 had high seropositivity yet prolonged viral shedding, which may be related to physiological deficiencies in gestational immunity, although we have not yet studied her T cell response. ${ }^{28}$

The primary strengths of this study are: the systematic investigation of all known prenatal COVID-19 cases in Singapore; comprehensive clinical analyses of maternal, neonatal and extra-fetal tissue samples to address the question of vertical transmission; and the inclusion of women affected in their first trimesters in this cohort, although perinatal data are still pending. The main limitations include the lack of universal screening to identify asymptomatic pregnant carriers, as this is not the current national policy given the low community transmission rate in Singapore, ${ }^{29}$ and the lack of extensive immunological investigations to understand the less aggressive disease progression in our cohort. Cumulative data are valuable in informing evolving maternal risk factors, viable treatment options for severe infection, and transplacental viral transmission.

\section{Conclusion}

The comprehensive reporting on all pregnant COVID19-infected patients managed in public hospitals in Singapore provides a complete review of maternal disease severity. Systematic assessment of prenatal and perinatal samples supports the low likelihood of motherchild transmission if the infection cleared well before delivery, with paired maternal-neonatal seropositivity suggesting transferred immunity. Our Singapore experience reflects a large series with the following findings: a generally low transmission rate; obese and older mothers being more prone to severe disease; no maternal mortality occurring in the hospital setting with close monitoring; and prompt interventional escalation 
made possible by a universal admission policy. Prolonged shedding shows variable duration of detectable viral RNA and is an important management consideration, however there is a lack of convincing evidence that this is predictive of actual infectivity. ${ }^{30}$

This case series is timely and directly addresses the specific concerns shared by pregnant women in Singapore psychologically affected by social distancing and other community measures implemented to curb the spread of COVID-19, ${ }^{31-33}$ even without contracting the infection. The data presented here is generally reassuring from the perspective of pregnancy outcomes, and makes a valuable contribution to inform general pandemic management with a focus on mental, as well as physical, well-being on pregnant and parturient women.

\section{REFERENCES}

1. Chen H, Guo J, Wang C, Luo F, et al. Clinical characteristics and intrauterine vertical transmission potential of COVID-19 infection in nine pregnant women: a retrospective review of medical records. Lancet 2020;395:809-15.

2. Dashraath P, Wong JLJ, Lim MXK, et al. Coronavirus disease 2019 (COVID-19) pandemic and pregnancy. Am J Obstet Gynecol 2020;222:521-31.

3. Lamouroux A, Attie-Bitach T, Martinovic J, Leruez-Ville M, Ville Y. Evidence for and against vertical transmission for severe acute respiratory syndrome coronavirus 2. Am J Obstet Gynecol 2020; 223:91.e1-e4.

4. Di Mascio D, Khalil A, Saccone G, Rizzo G, Buca D, Liberati M, et al. Outcome of coronavirus spectrum infections (SARS, MERS, COVID-19) during pregnancy: a systematic review and metaanalysis. Am J Obstet Gynecol MFM 2020;2:100107.

5. Wu JT, Leung K, Bushman M, Kishore N, Niehus R, de Salazar PM, et al. Estimating clinical severity of COVID-19 from the transmission dynamics in Wuhan, China. Nat Med 2020;26:506-10.

6. Blitz MJ, Grünebaum A, Tekbali A, Bornstein E, Rochelson B, Nimaroff $\mathbf{M}$, et al. Intensive care unit admissions for pregnant and non-pregnant women with coronavirus disease 2019. Am J Obstet Gynecol 2020;223:290-91.

7. Amorim MMR, Soligo Takemoto ML, Fonseca EBD. Maternal deaths with coronavirus disease 2019: a different outcome from low- to middleresource countries? Am J Obstet Gynecol 2020;223:298-99.

8. Hantoushzadeh S, Shamshirsaz AA, Aleyasin A, Seferovic MD, Aski SK, Arian SE, et al. Maternal death due to COVID-19. Am J Obstet Gynecol 2020;223:109.e1-e16.

9. Mahyuddin AP, Kanneganti A, Wong JJL, Dimri PS, Su LL, Biswas A, et al. Mechanisms and evidence of vertical transmission of infections in pregnancy including SARS-CoV-2s. Prenat Diagn 2020. doi: $10.1002 /$ pd.5765.
10. Hu X, Gao J, Luo X, Feng L, Liu W, Chen J, et al. Severe acute respiratory syndrome coronavirus 2 (SARS-CoV-2) vertical transmission in neonates born to mothers with coronavirus disease 2019 (COVID-19) pneumonia. Obstet Gynecol 2020;136:65-7.

11. Poljak M, Korva M, Knap Gašper N, Fujs Komloš K, Sagadin M, Uršič T, et al. Clinical evaluation of the cobas SARS-CoV-2 test and a diagnostic platform switch during 48 hours in the midst of the COVID-19 pandemic. J Clin Microbiol 2020;58:e00599-20.

12. Academy of Medicine Singapore. Position statement from the National Centre for Infectious Diseases and the Chapter of Infectious Disease Physicians: period of infectivity to inform strategies for de-isolation for COVID-19 patients. Published 23 May 2020. Available at: https:// www.ams.edu.sg/view-pdf.aspx?file=media\%5C5556 fi 331.pdf\&ofil $\mathrm{e}=$ Period + of + Infectivity + Position + Statement $+($ final $)+23-5-20+(\log$ os $)$. pdf. Accessed on 1 Aug 2020.

13. Breslin N, Baptiste C, Gyamfi-Bannerman C, Miller R, et al. Coronavirus disease 2019 infection among asymptomatic and symptomatic pregnant women: two weeks of confirmed presentations to an affiliated pair of New York City hospitals. Am J Obstet Gynecol MFM 2020;2:100118.

14. Pierce-Williams RAM, Burd J, Felder L, Khoury R, Bernstein PS, Avila $\mathrm{K}$, et al. Clinical course of severe and critical coronavirus disease 2019 in hospitalized pregnancies: a United States cohort study. Am J Obstet Gynecol MFM 2020;2:100134.

15. Hirshberg A, Kern-Goldberger AR, Levine LD, Pierce-Williams R, Short WR, Parry S, et al. Care of critically ill pregnant patients with coronavirus disease 2019: a case series. Am J Obstet Gynecol 2020;223:286-90

16. Xu K, Chen Y, Yuan J, Yi P, Ding C, Wu W, et al. Factors associated with prolonged viral RNA shedding in patients with coronavirus disease 2019 (COVID-19). Clin Infect Dis 2020;71:799-806.

17. Zhang W, Du RH, Li B, Zheng XS, Yang XL, Hu B, et al. Molecular and serological investigation of 2019-nCoV infected patients: implication of multiple shedding routes. Emerg Microbes Infect 2020;9:386-89.

18. Shanes ED, Mithal LB, Otero S, Azad HA, Miller ES, Goldstein JA. Placental pathology in COVID-19. Am J Clin Pathol 2020;154:23-32.

19. Tan TY, Yeo GS. Intrauterine growth restriction. Curr Opin Obstet Gynecol 2005;17:135-42.

20. COVID-19 dashboard by the Center for Systems Science and Engineering (CSSE) at Johns Hopkins University. Available at: https://coronavirus.jhu.edu/map.html. Accessed on 1 Aug 2020.

21. Algarroba GN, Rekawek P, Vahanian SA, Khullar P, Palaia T, Peltier MR, et al. Visualization of severe acute respiratory syndrome coronavirus 2 invading the human placenta using electron microscopy. Am J Obstet Gynecol 2020;223:275-78.

22. Calomeni E, Satoskar A, Ayoub I, Brodsky S, Rovin BH, Nadasdy T. Multivesicular bodies mimicking SARS-CoV-2 in patients without COVID-19. Kidney Int 2020;98:233-34.

23. Zeng H, Xu C, Fan J, Tang Y, Deng Q, Zhang W, et al. Antibodies in infants born to mothers with COVID-19 pneumonia. JAMA 2020;323:1848-9.

24. Patanè L, Morotti D, Giunta MR, Sigismondi C, Piccoli MG, Frigerio L, et al. Vertical transmission of coronavirus disease 2019: severe acute respiratory syndrome coronavirus 2 RNA on the fetal side of 
the placenta in pregnancies with coronavirus disease 2019-positive mothers and neonates at birth. Am J Obstet Gynecol 2020;2:100145.

25. Vivanti AJ, Vauloup-Fellous C, Prevot S, Zupan V, Suffee C, Do Cao J, et al. Transplacental transmission of SARS-CoV-2 infection. Nat Commun 2020;11:3572.

26. Groß R, Conzelmann C, Müller JA, Stenger S, Steinhart K, Kirchhoff $\mathrm{F}$, et al. Detection of SARS-CoV-2 in human breastmilk. Lancet 2020;395:1757-8.

27. Zhao J, Yuan Q, Wang H, Liu W, Liao X, Su Y, et al. Antibody responses to SARS-CoV-2 in patients with novel coronavirus disease 2019. Clin Infect Dis 2020;2020;71:2027-34.

28. Le Bert N, Tan AT, Kunasegaran K, Tham CYL, Hafezi M, Chia A, et al. SARS-CoV-2-specific $\mathrm{T}$ cell immunity in cases of COVID-19 and SARS, and uninfected controls. Nature 2020; 584:457-62.
29. Sun Y, Koh V, Marimuthu K, Ng OT, Young B, Vasoo S, et al; National Centre for Infectious Diseases COVID-19 Outbreak Research Team. Epidemiological and clinical predictors of COVID-19. Clin Infect Dis 2020;71:786-92.

30. Widders A, Broom A, Broom J. SARS-CoV-2: The viral shedding vs infectivity dilemma. Infect Dis Health 2020;25:210-5.

31. Ng QJ, Koh KM, Tagor S, et al. Perception and Feelings of Antenatal Women during COVID-19 Pandemic: A Cross-Sectional Survey. Ann Acad Med Singap 2020;49:543-52.

32. Chen JI, Yap JC, Hsu LY, et al. COVID-19 and Singapore: From Early Response to Circuit Breaker. Ann Acad Med Singap 2020; 49:561-72.

33. Tan THY, Toh M, Vasoo S, et al. Coronavirus Disease 2019 (COVID-19): The Singapore Experience. A Review of the First Eight Months. Ann Acad Med Singap 2020;49:764-78. 